
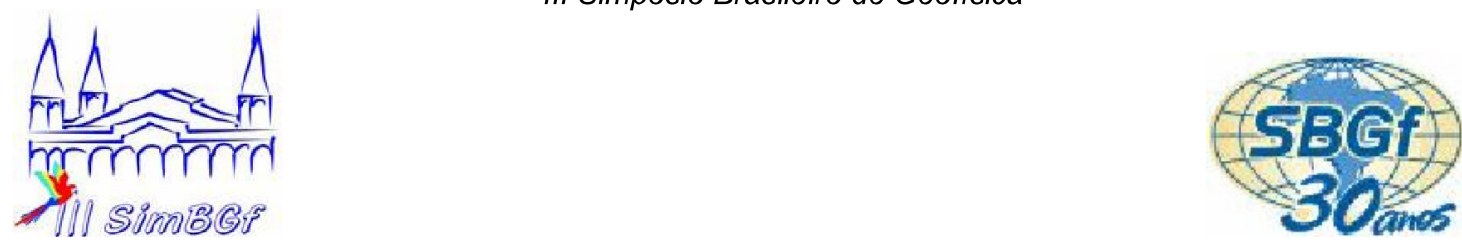

\title{
Desenvolvimento de um heliômetro e sua aplicação em conjunção com medições geomagnéticas
}

Eugênio Reis Neto*, Observatório Nacional/MCT, Brasil

Victor de Amorim d'Ávila, Observatório Nacional, MCT and IGEO/UERJ, Brasil

Alexandre Humberto Andrei, Observatório Nacional/MCT \& Observatório do Valongo/UFRJ, Brasil

Jucira Lousada Penna, Observatório Nacional/MCT, Brasil

Andrés Reinaldo Rodriguez Papa, Observatório Nacional/MCT \& IF/UERJ, Brasil

Sérgio Calderari Boscardin, Observatório Nacional/MCT, Brasil

Luiz Carlos Oliveira, Observatório Nacional/MCT \& Grupo de Astronomia NGC-51, Brasil

Kennedy de Ávila, Observatório Nacional/MCT, Brasil

Copyright 2008, SBGf - Sociedade Brasileira de Geofisica

Este texto foi preparado para a apresentação no III Simpósio Brasileiro de Geofísica, Belém, 26 a 28 de novembro de 2008. Seu conteúdo foi revisado pelo Comitê Técnico do III SimBGf mas não necessariamente representa a opinião da SBGf ou de seus associados. É proibida a reprodução total ou parcial deste material para propósitos comerciais sem prévia autorização da SBGf.

\section{Abstract}

The objective of this work is to develop, build and test an instrument, based on the heliometric method, to measure with great precision, in all heliolatitudes, the solar disk and its asphericity, as well as its variations as a function of time. Its possible use simultaneously with other measures of solar activity and in comparison of the survey of geomagnetic activity is also considered. Four promising methods to provide scientific results were tested. The best method doubles the image of the Sun by means of a parabolic mirror, cut and reassembled with angular displacement. The program of analysis of the images is being developed and codified in Borland Delphi. Hundreds of images were analyzed and the results have shown an accuracy of 0.09 seconds of arc for the measurement of the solar diameter.

\section{Introdução}

O Heliômetro é um instrumento dedicado a medições de grande precisão do diâmetro solar. A técnica de medição consiste no desdobramento da imagem do disco solar em duas, por uma distância angular conhecida e a posterior determinação do diâmetro solar através da medida da separação entre bordos opostos adjacentes.

Este tipo de instrumento foi primeiramente proposto em 1675 pelo astrônomo dinamarquês Ole Rømer e construído em 1748 pelo astrônomo francês Pierre Bouguer, que montou um telescópio com duas objetivas, que foram colocadas lado a lado $e$ que, conseqüentemente, formavam duas imagens dos objetos observados. Através de uma escala colocada no foco em comum, media-se a distância de contato entre os limbos solares. Mas tarde, em 1754, John Dollond sugeriu o corte da objetiva, como forma de duplicar a imagem. Em 1838, a partir das observações heliométricas, Friedrich Bessel conseguiu, pela primeira vez, determinar a paralaxe da estrela 61 Cygni (Zeilik \& Gregory,1998).
O monitoramento do diâmetro solar é atualmente realizado pelo Heliômetro do Pic-du-Midi (Rosch, J., Yerle, R. 1983; Rösch, J. et al., 1996), pelo Astrolábio de Calern (Delmas \& Laclare, 2002), que juntamente com o Astrolábio do Observatório Nacional, lidera a Rede de Monitoramento Solar (Andrei et al., 2004), pelo Monitor de Imagens Solares de Calern, pelo telescópio Solar de Locarno (Wittmann \& Bianda, 2000). Entre as medidas espaciais estão as obtidas com os satélites SOHO (Kuhn et al., 2004) e RHESSI (Fivian et al., 2007). Espera-se para 2009 o lançamento do Micro-satélite Picard (Thuillier, 2003). Este satélite fará medidas simultâneas da irradiação solar total, da forma do Sol, irradiação de UV e suas variabilidades, que quando correlacionadas com as observações semelhantes feitas em solo, servirão para calibrar as mesmas e aperfeiçoar modelos atmosféricos.

\section{O Princípio Observacional}

- Diferentemente do Astrolábio Solar do Observatório Nacional (Jilinski et al., 1998; Penna et al., 2002), que mede o diâmetro do Sol pelo método dinâmico das alturas iguais (computando o tempo de trânsito do disco solar através de uma determinada altura), o Heliômetro fará medidas astrométricas do disco solar, diretamente das imagens geradas por uma câmera CCD.

- Duplicando-se a imagem do Sol, por qualquer método óptico-instrumental, não há necessidade de fazer com que todo o disco caiba dentro da matriz do CCD (figura 1), que poderá ser composta por pixels com a menor dimensão possível, aumentando a resolução espacial.

- As imagens devem ser deslocadas e separadas por uma distância angular pouco maior que $0,5^{\circ}$. Esta separação corresponde à distância angular entre os centros dos discos. Ela será função do instrumento e será sempre conhecida com precisão. Bordos opostos do Sol (pontos $A$ e B, da figura 1) estarão próximos um ao outro. A medida da distância mínima entre estes bordos, subtraída da distância entre os centros, determina o diâmetro solar nesta heliolatitude. 
- Girando-se o instrumento em seu eixo óptico, será possível fazer medidas em todas as heliolatitudes, numa mesma sessão observacional.

- Também não há necessidade de que o instrumento tenha acompanhamento, pois a passagem das imagens dará informações como a escala de placa, por exemplo.

- O número de imagens para a análise só será limitado pelo espaço disponível para armazená-las, para o que, metodologia específica deve ser desenvolvida.

- A precisão das medidas baseia-se na estabilidade dimensional dos materiais usados na fabricação do instrumento: fibra de carbono (estrutura), CCZ-HS (espelho), além da regulagem da temperatura da cúpula e de todo o instrumento, bem como em técnicas de monitoramento por auto-colimação.

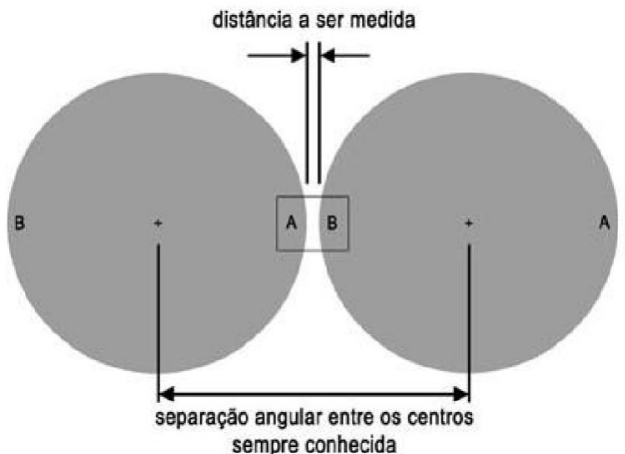

Figura 1 - Esquema do princípio observacional do disco solar. $O$ retângulo no centro corresponde à matriz do CCD. Bordos diametralmente opostos (A e B) ficam próximos um do outro.

\section{Detalhes Experimentais}

As quatro técnicas heliométricas testadas foram:

- Uso de prismas montados após a ocular do instrumento (mesmo princípio duplicador usado em microscópios binoculares);

- Disposição de dois espelhos planos, na frente da objetiva do instrumento, contidos em planos que formam um diedro;

- Corte da objetiva ao longo de seu diâmetro (método clássico);

- Corte de um espelho telescópico ao longo de seu diâmetro.

Para cada uma das técnicas, um protótipo foi construído, sendo que esta última técnica foi a escolhida por sua simplicidade (mínimo de superfícies ópticas envolvidas), e acromatismo perfeito. $O$ instrumento foi projetado para possuir distância focal de $1 \mathrm{~m}$.

No primeiro heliômetro refletor construído, as metades do espelho foram afastadas linearmente, de modo perpendicular ao corte, mantendo os eixos ópticos paralelos entre si. Numa segunda montagem, as metades do espelho foram deslocadas angularmente, com o eixo de rotação perpendicular ao corte.

A câmera CCD utilizada foi a Philips PCVC840K ToUcam, que gerou imagens no formato BMP, com $480 \times 640$ pixels quadrados de $5,6 \mu \mathrm{m}$ de lado.
A medida da distância mínima entre os bordos opostos adjacentes é obtida primeiramente com a identificação dos bordos dos discos solares, através da definição clássica: a identificação das coordenadas dos pontos de inflexão da função luminosidade ao longo de cada linha da imagem. Na figura 2 vê-se um exemplo desta abordagem. A curva do centro corresponde à função luminosidade de uma das linhas da imagem. Abaixo dela, a mesma função alisada através de uma média corrida ponderada, e acima, a derivada numérica da curva alisada. As curvas foram deslocadas verticalmente para melhor visualização. As abscissas dos pontos de inflexão da função de luminosidade da curva alisada serão aquelas onde a derivada primeira passar por extremos um mínimo, para a imagem do disco solar da esquerda, e um máximo, para o da direita.

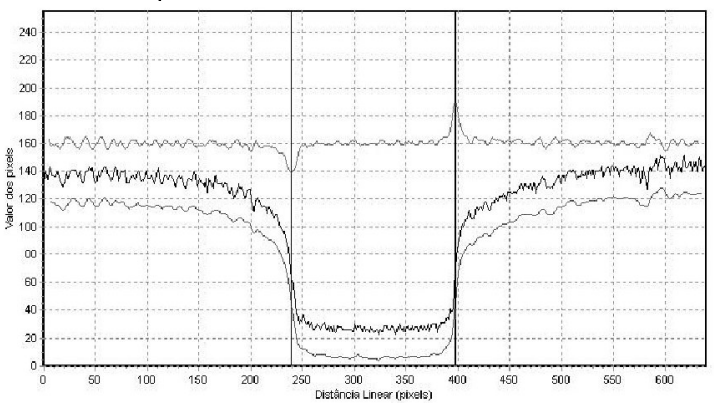

Figura 2 - Função luminosidade de uma das linhas da imagem. As linhas verticais cheias indicam as abscissas dos pontos de inflexão da curva alisada.

Depois de identificar os dois conjuntos de pontos de inflexão em todas as linhas da imagem, o programa encontra as coordenadas dos centros e os raios dos discos solares, em primeira aproximação, por ajuste de funções parabólicas.

Com estes pontos de partida, duas circunferências são ajustadas por mínimos quadrados, eliminando-se os pontos que estiverem além de $3 \sigma$ do ajuste, corrigindo os valores iniciais e calculando a incerteza das medidas.

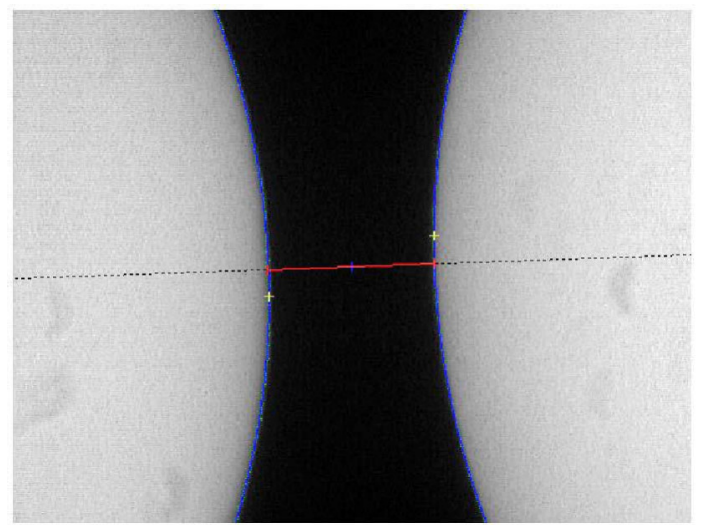

Figura 3 - Imagem heliométrica do Sol. As cruzes indicam os pontos mais extremos dos discos. A linha tracejada representa a reta que une os centros dos discos e o segmento de reta é a distância mínima encontrada. 
A reta que une os centros dos discos é ajustada e as coordenadas dos pontos onde esta reta faz interseção com as circunferências são calculadas. A separação entre estes pontos representa a menor distância entre os discos (Figura 3).

No exemplo da imagem na Figura 3 , a incerteza da medida da distância mínima e de $\pm 0,06$ pixel. Como a distância entre os centros é função do instrumento e será sempre conhecida, o diâmetro do Sol será conhecido com esta mesma ordem de precisão.

\section{Resultados}

O cálculo da distância mínima entre os bordos dos discos não necessita que o Heliômetro tenha motor de acompanhamento. $\mathrm{O}$ instrumento é apontado para o Sol e ajustado de forma que os dois discos estejam no campo. O Telescópio permanece, então, parado enquanto as imagens dos discos do Sol atravessam a matriz do CCD. O sistema de aquisição captura uma imagem a cada segundo, numa média de 50 por passagem. A direção desta passagem, em relação às linhas do $C C D$, depende do ângulo entre a direção do movimento aparente do Sol no céu e a direção do deslocamento entre as imagens duplicadas.

No começo de 2007 foram realizadas duas sessões com o espelho na configuração angular. As sessões foram realizadas no Rio de Janeiro, com o céu muito limpo e sem vento, tomando-se o cuidado de esperar o instrumento estabilizar-se, após o reposicionamento para a passagem seguinte. Os resultados estão na Tabela 1.

Tabela 1: Valores médios e dispersão das distâncias mínimas entre os bordos das imagens obtidas com a configuração angular. Os valores estão em escala de pixels.

\begin{tabular}{|c|c|c|c|c|}
\hline $\begin{array}{c}\text { Sessão } \\
\text { (dia) }\end{array}$ & passagem & $\begin{array}{c}\mathrm{n}^{\circ} \mathrm{de} \\
\text { imagens }\end{array}$ & $\begin{array}{c}\text { distância } \\
\text { mínima } \\
\text { (pixel) } \\
\end{array}$ & $\begin{array}{c}\sigma \\
\text { (pixel) }\end{array}$ \\
\hline \multirow{4}{*}{$\begin{array}{c}8 \mathrm{de} \\
\text { fevereiro }\end{array}$} & $1^{\circ}$ & 49 & $94,01 \pm 0,10$ & 0,71 \\
\hline & $2^{\circ}$ & 30 & $93,68 \pm 0,24$ & 1,31 \\
\hline & $3^{\circ}$ & 52 & $94,21 \pm 0,18$ & 1,27 \\
\hline & $4^{\circ}$ & 56 & $93,95 \pm 0,11$ & 0,81 \\
\hline total & 4 & 187 & $94,00 \pm 0,08$ & 1,03 \\
\hline \multirow{2}{*}{$\begin{array}{c}9 \mathrm{de} \\
\text { fevereiro }\end{array}$} & $1^{\circ}$ & 66 & $93,65+0,09$ & 0,75 \\
\hline & $2^{\circ}$ & 48 & $93,89 \pm 0,11$ & 0,76 \\
\hline total & 2 & 114 & $93,75 \pm 0,07$ & 0,76 \\
\hline
\end{tabular}

A Figura 4 mostra a variação da distância mínima para as duas passagens do dia 9 . O ajuste linear das medidas também é mostrado.

Os coeficientes lineares dos ajustes são

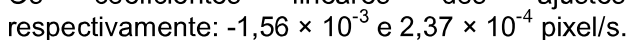

A evolução temporal da distância mínima e da distância entre os centros, para estas duas passagens é vista na Figura 5. A primeira em vermelho e a última em azul. Não existe correlação significativa entre estes dois conjuntos de valores $(\cong 0,17$, a $99 \%)$. Os eixos verticais estão na mesma escala, para comparação.

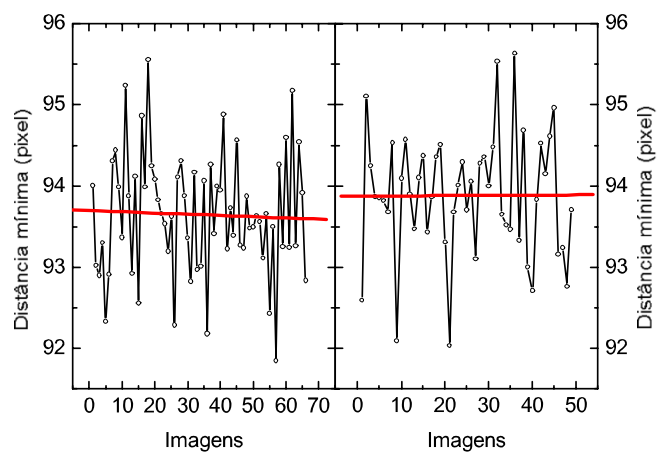

Figura 4: Seqüência de medidas da distância mínima das passagens do dia 9 de fevereiro.

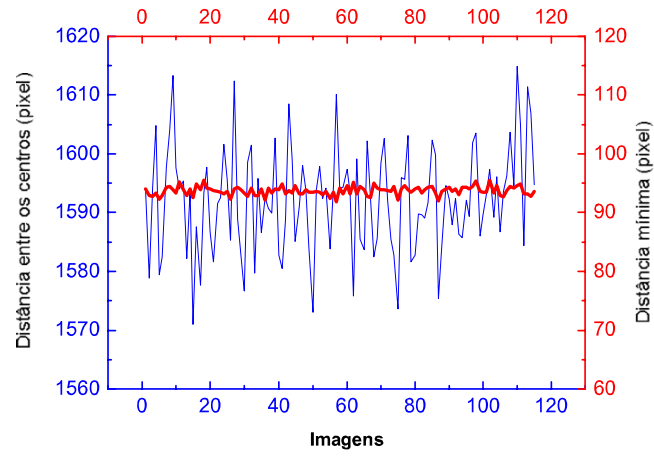

Figura 5: Comparação entre as evoluções temporais da distância mínima e da distância entre os centros. A dispersão dos valores da distância entre os centros é de $\sim 9,0$ pixels.

A boa estabilidade das imagens desta última sessão permitiu demonstrar o cálculo da escala de placa da montagem. Foram utilizadas as coordenadas do ponto médio da distância mínima entre os dois bordos, do primeiro ao último frame, da segunda passagem da sessão do dia 9 de fevereiro de 2007. Através do instante de aquisição de cada imagem, foi possível o cálculo da velocidade de passagem deste ponto pela matriz do CCD.

A velocidade de passagem deste ponto médio foi comparada à velocidade média aparente do Sol $(\approx 15 " / s)$. O que resultou numa escala de placa de $\approx 1 ", 32 /$ pixel, o que para uma incerteza na medida de 0,07 pixel corresponde a precisão de 0",09 na medida.

\section{Discussão e Conclusão}

Os cuidados instrumentais na hora da aquisição diminuíram o viés instrumental, a ponto de fazer com que a incerteza da medida da distância mínima entre os 
bordos ficasse em torno de 0,1 pixel e a dispersão, abaixo de 1,0 pixel. A pequena tendência linear da distância mínima (Figura 4) indica que a configuração do espelho permaneceu relativamente estável durante as passagens, no entanto demonstrando a utilidade de partes ópticas termicamente invariáveis.

A não correlação da distância entre os centros dos discos solares e a distância mínima entre eles, demonstra a estabilidade do método utilizado, sendo esta última, o melhor parâmetro para as medidas heliométricas. A diferença entre a dispersão destes valores $(9,0$ versus 0,76 pixel) parece indicar que para a primeira, as flutuações se devem à limitação do ajuste das circunferências (não linear e muito sensível ao número de pontos utilizados), enquanto que para a última, as flutuações se devem a ruídos observacionais.

A incerteza de 0,07 pixel na determinação da distância mínima entre os bordos traduz-se numa precisão de 0",09 na medida alcançada por este protótipo. A dispersão dos valores das medidas foi de $\sim 1 "$.

A utilização de uma câmera CCD com pixels quadrados de 4,4 micra, em uma matriz de 1,6k x 1,2k elementos, corresponderá a uma escala de placa de $0 ", 8 / p x$, para a distância focal de $1 \mathrm{~m}$, equivalendo à precisão de 40 mas (milisegundo de arco) em cada linha do CCD. Assim, atinge-se a amostragem permitida pelo seeing diurno em uma única medida.

Este instrumento representa um importante ganho observacional em relação ao instrumento atualmente instalado no campus do Observatório Nacional (tabela 2).

Tabela 2: Comparação entre o Astrolábio e o Heliômetro

\begin{tabular}{c|c|c} 
& $\begin{array}{c}\text { Astrolábio } \\
\text { Solar }\end{array}$ & Heliômetro \\
\hline $\begin{array}{c}\text { Precisão de uma } \\
\text { medida isolada }\end{array}$ & $0,5^{\prime \prime}$ & $0,1^{\prime \prime}<p<0,05^{\prime \prime}$ \\
\hline Acurácia & $0,005^{\prime \prime} /$ ano & $0,005^{\prime \prime} / \mathrm{mês}$ \\
\hline $\begin{array}{c}\text { Quantidade de } \\
\text { medidas }\end{array}$ & até 30/dia & até $10.000 /$ dia \\
\hline Recobrimento em heliolatitudes & semi-anual & diário \\
\hline
\end{tabular}

De 1997 até 2003 o Astrolábio Solar do Observatório Nacional fez mais de 20.000 observações do diâmetro solar. Os resultados da análise desta longa série mostram variações do diâmetro do Sol ao longo deste período, assim como diferentes valores por heliolatitude observada (Reis Neto et al., 2003), e que existem correlações entre o diâmetro do Sol e os parâmetros de atividade solar (Boscardin et al., 2008).

Com o Heliômetro em pleno funcionamento, o volume de dados a serem analisados será muito maior (vide Tabela 2), abrindo a possibilidade de estudos mais completos sobre a correlação entre o diâmetro solar e outros estimadores da atividade solar em geral (Andrei et al., neste Simpósio), e em particular, medições geomagnéticas (Dias et al., neste Simpósio).

\section{Agradecimentos}

Os autores agradecem o apoio financeiro da FAPERJ, CNPq e FINEP.

\section{Referências}

Andrei, A.H., Penna, J.L., Papa, A.R.R., d'Ávila, V.A., Reis Neto, E., Boscardin, S.C., 2008. III Simpósio Brasileiro de Geofísica.

Andrei, A. H., Boscardin, S. C., Chollet, F., Delmas, C., Golbasi, O., Jilinski, E. G., Kiliç, H., Laclare, F., Morand, F., Penna, J. L. \& Reis Neto, E., 2004. Comparison of CCD astrolabe multi-site solar diameter observations. Astronomy \& Astrophysics, 427: pp 717-723.

Boscardin, S. C., Andrei, A. H., Penna, J. L. and Reis Neto, E. \& d'Ávila, V.A., 2008. Correlation Analysis of Solar Semi-Diameter Results. Astronomy \& Astrophysics - submitted.

Chris Lord, 2000. History \& Development of the DividedLens Micrometer \& Calcite Double-Image Micrometer. Publications of Brayebrook Observatory.

Delmas, C., \& Laclare, F. 2002, Solar Physics, 209: pp 391.

Dias, V.H.A., Figueiredo, I., Penna, J.L., Andrei, A.H., Papa, A.R.R., 2008. III Simpósio Brasileiro de Geofísica.

Fivian, M. D., Hudson, H. S., Lin, R. P., Zahid, H. J., 2007. American Geophysical Union, Fall Meeting 2007, abstract \#SH53A-1076.

Jilinski, E. G., Puliaev, S. P., Penna, J. L., Andrei, A. H., Sinceac, V., Chollet, F. \& Delmas, C., 1998. Astronomy and Astrophysics Supplement, 130: pp 317-321.

Kuhn, J. R., Bush, R. I., Emilio, M., \& Scherrer, P. H., 2004. The Astrophysical Journal, 613: pp 1241-1252.

Penna, J. L., Jilinski, E. G., Andrei, A. H., Puliaev, S. P. \& Reis Neto, E., 2002. Astronomy and Astrophysics, 384: pp 650-653.

Reis Neto, E., Andrei, A. H., Penna, J. L., Jilinski, E. G. \& Puliaev, S. P, 2003. Solar Physics, 212: pp 7-21.

Rösch, J., Yerle, R., 1983. Solar Physics, 82, pp 139-150.

Rösch, J., Rozelot, J. P., Deslandes, H. \& Desnoux, V.,1996. Solar Physics, Volume 165: pp.1-11.

Thuillier, G., Joukoff, A., \& Schmutz, W., 2003. Solar variability as an input to the Earth's environment. The PICARD mission, Proceeding of the ISCS Symposium. ESA SP-535: pp. 251-257.

Wittmann, A. D.\& Bianda, M., 2000. The solar cycle and terrestrial climate, Solar and space weather Euroconference. Proceedings of the 1st Solar and Space Weather Euroconference. ESA SP, 463: pp113.

Zeilik, Michael A. \& Gregory, Stephan A., 1998. Introductory Astronomy \& Astrophysics, Saunders College Publishing, 4th ed.: pp 44. 